\title{
Wavelet-based higher-order neural networks for mine detection in thermal IR imagery
}

\author{
Brian A. Baertlein and Wen-Jiao Liao \\ The Ohio State University ElectroScience Laboratory \\ 1320 Kinnear Road, Columbus, OH 43212
}

\begin{abstract}
An image processing technique is described for the detection of mines in IR imagery. The proposed technique is based on a third-order neural network, which processes the output of a wavelet packet transform. The technique is inherently invariant to changes in signature position, rotation and scaling. The well-known memory limitations that arise with higher-order neural networks are addressed by (1) the data compression capabilities of wavelet packets, (2) projections of the image data into a space of similar triangles, and (3) quantization of that "triangle space." Using these techniques, image chips of size $28 \times 28$, which would require $O\left(10^{9}\right)$ neural net weights, are processed by a network having $O\left(10^{2}\right)$ weights. ROC curves are presented for mine detection in real and simulated imagery.
\end{abstract}

Keywords: land mines, IR imagery, wavelets, neural networks

\section{INTRODUCTION}

It has long been recognized that surface-laid and buried mines can be detected using infrared (IR) sensors. ${ }^{1}$ Although significant strides have been made in both the sensors and image processing, detection of mines in natural clutter remains a challenging problem. The challenges arise, in part, because of a large number of unknown random signature variables including amplitude, contrast polarity, rotation, and size.

There exist several techniques for dealing with unknown signature variables. When a sufficiently accurate model for the signature exists and the distribution of the unknown variables is known, one can perform an integration over the variables, leading to an optimal Bayesian detector. ${ }^{2}$ For many IR targets (especially buried mines), such models are impractical since the signature is strongly affected by the environment (surface topology, solar illumination history, etc.), and the number of unknown variables becomes excessive.

As an alternative, one can use a maximum likelihood technique to estimate the unknown variables, leading to a generalized likelihood ratio test (GLRT). Detection is then based on the error between the model (using the estimated parameters) and the data. In a recent work ${ }^{3}$ a nonlinear optimization technique was used to estimate parameters for an empirical signature model. These parameters and the resulting model error were both used in a classifier to detect the presence of the mine. That technique, which we refer to as an "estimator-classifier" (by analogy with the "estimator-correlator" technique used for random Gaussian-distributed signals), is capable of better performance than the GLRT, since it makes direct use of the model parameters. A significant limitation of all such approaches is the extensive processing required for nonlinear optimization. Because imaging systems tend to produce massive data sets, computational efficiency is a concern.

In this work we emphasize detection of surface mines and mine fields. An important aspect of these targets is that they tend to have a small number of simple shapes (round, square, etc.). Thus, for surface mines and some buried mines, the signature of the mine is largely known with the exception of a translation (due to its position in the image), a scale factor (due to the image resolution, the size of the mine, or the burial depth), and a rotation (for non-circular mines). In IR detection of mine fields the (near) regular spatial pattern of the mines is an important discriminant. The pattern, however, will have an unknown rotation and translation, and it may vary in scale as a result of changes in image resolution or mine-laying practice.

Detection of objects with unknown position, scale and rotation (PSR) is an important general topic, and the subject has been explored by many researchers. It is well known that the magnitude of the two-dimensional Fourier transform $\mathcal{F}$ is invariant to translational shifts. Mehanian and $\mathrm{Rak}^{4}$ showed that a log-polar mapping of the Fourier

Corresponding author: B.A.B. (614) 292-0076 (voice), (614) 292-7297 (fax), baertlein.1@osu.edu 
transform magnitude could produce a representation that was position, scale and rotation invariant (PRSI). A limitation of this approach is that the polar coordinates must be defined with respect to a "stable" point in the image. The centroid of the signature has been used in that role, but the centroid is an unreliable reference for targets in a highly cluttered environment.

The magnitude of the Mellin transform $\mathcal{M}$ is invariant to changes in scale, and this fact has been used to develop scale-invariant detection systems. During the 1970s it was observed by several groups ${ }^{5-8}$ that the combination of $|\mathcal{F}|$ followed by $|\mathcal{M}|$, the so-called Fourier-Mellin (FM) transform, could be used for this purpose. The use of wavelets for scale invariance has been investigated by several groups. Baertlein and Casey ${ }^{9}$ developed an analog of the FourierMellin transform noted above, in which the continuous wavelet transform of the Fourier transform modulus was computed. Szu et al. ${ }^{10}$ used a similar technique in which the continuous wavelet transform was computed optically. Yoon et al. ${ }^{11}$ used the CWT of the target boundary to obtain a scale-invariant representation for image recognition. Although all of these approaches provide translation and scale invariance, they are not rotationally invariant.

In this paper we propose a technique that employs higher-order neural networks to perform PSRI detection. The memory required by a higher-order network can be significant, but the image decomposition and data compression capabilities of the wavelet transform are used to mitigate this problem.

The paper is organized in three major sections. Higher-order neural networks are described in Section 2. A wavelet packet transform is required in this work, and that topic is described in Section 3. Classification results for real and simulated imagery are provided in Section 4. Concluding remarks appear in Section 5.

\section{NEURAL NETWORKS AND PSR INVARIANCE}

Several approaches have been developed for incorporating PSR invariance into neural networks. ${ }^{12}$ Feed-forward multilayer perceptrons (FFMLPs) can be trained to recognize signatures that have been shifted, rotated, and scaled, but the amount of training data required is large, and accuracy is limited. ${ }^{13,14}$ The neocognitron ${ }^{15,16}$ is a novel neural network architecture that attempts to achieve PSRI performance by mimicking the structure of the human visual system. Modest deformations and small rotations (at most a few degrees) are tolerated in the input, but rotational invariance over a wider range of angles has not been demonstrated.

Higher-order neural networks (HONNs) offer perhaps the best approach to the PSRI problem. The theoretical nature of invariant pattern classification has been discussed by Minsky and Papert. ${ }^{17}$ Lee et al. ${ }^{18}$ describe the concept of correlations among higher-order products in neural networks. Giles and Maxwell ${ }^{19}$ showed that by a suitable sharing of weights one could structurally induce translation invariance in both associative memories and FFMLPs while simultanteously reducing the number of weights to be learned. The key benefit of such networks is that the translation invariance property need not be learned from data. Examples acquired at a fixed position are sufficient to train the network to recognize shifted data. A combination of HONNs and log-polar mappings has also been investigated. ${ }^{20}$

The computation performed by a higher-order neuron output is straightforward. Consider an image of size $N \times N$ in which the pixels $x_{i j}$ have been indexed as $x_{i_{n} j_{n}}, n=1,2, \ldots, N^{2}$. The higher-order neuron output has the form

$$
y=f\left(\sum_{n=1}^{N^{2}} x_{i_{n} j_{n}} w_{n}^{(1)}+\sum_{n=1}^{N^{2}} \sum_{m=1}^{N^{2}} x_{i_{n} j_{n}} x_{i_{m} j_{m}} w_{n m}^{(2)}+\sum_{n=1}^{N^{2}} \sum_{m=1}^{N^{2}} \sum_{k=1}^{N^{2}} x_{i_{n} j_{n}} x_{i_{m} j_{m}} x_{i_{k} j_{k}} w_{n m k}^{(3)}+\cdots\right)
$$

where $f(\cdot)$ is the activation function (nominally a sigmoid-like function) and the weights are given by $w_{n}^{(1)}, w_{n m}^{(2)}$ and $w_{n m k}^{(3)}$ for first, second, and third orders, respectively. As an example, the architecture of a third-order neuron is shown in Figure 1.

The linear sum is analogous to the output of a conventional neuron. The sum of second-order products involves correlations, and the third-order sum involves bicorrelations. The higher-order products explicitly provide the nonlinear relations on which the network operates. Note that this is a single-layer network, i.e., the output neuron is connected directly to the input data. As a result, training may be performed with the simple Widrow-Hoff algorithm. For example, for the third-order weights, the update equation for a network that produced output $s$ for a desired output $t$ is

$$
\Delta w_{n m k}^{(3)}=(t-s) x_{i_{n} j_{n}} x_{i_{m} j_{m}} x_{i_{k} j_{k}}
$$




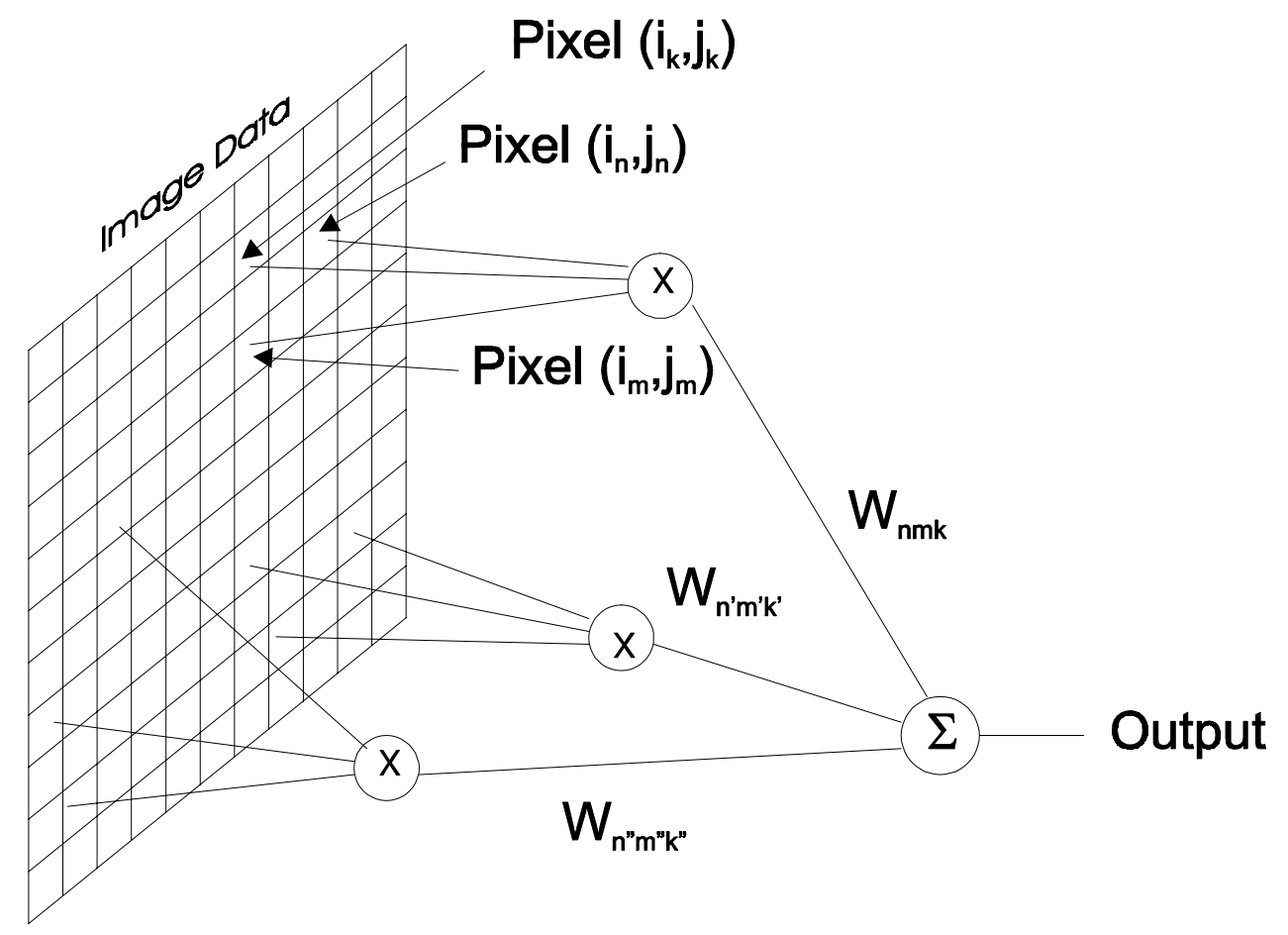

Figure 1. The architecture of a third-order neural network. Only three weight connections are shown.

Spirkovska and Reid ${ }^{21}$ showed that a weight-sharing technique could be used to produce second-order FFMLPs with translation and scale invariance. Such a HONN is useful for detection of rotationlly symmetric signatures, which comprise most surface mines and essentially all buried mines at depths greater than a few inches. To achieve this capability we equate weights corresponding to line pairs whose lengths have a similar ratio. Explicitly, the equivalence we require is

$$
w_{n m}^{(2)}=w_{k \ell}^{(2)} \text { if }\left|i_{n}-i_{m}\right| /\left|j_{n}-j_{m}\right|=\left|i_{k}-i_{\ell}\right| /\left|j_{k}-j_{\ell}\right|
$$

This equivalence maps greatly reduces the number of weights and the training data requirements for the network.

Recognition of objects that have been simultaneously scaled, rotated and translated requires a third-order network, ${ }^{21}$ the memory and computational requirements of which are substantial. For an image of size $N \times N$, a network or order $M$ requires $N^{2 M}$ words to store the network weight values. For image chips of size $N=32$ and a third-order HONN $(M=3)$, this leads to a requirement for $1.1 \times 10^{9}$ words of storage. For a focal plane with $128 \times 128$ pixels, the requirement grows to $4.4 \times 10^{12}$ words.

The weight sharing required for PSRI performance in a third-order HONN mitigates this need to a degree and proceeds as follows: ${ }^{22,21}$ Triads of points share a weight if they can be related by a translation, rotation and scaling. Thus, pixels triads that form geometrically similar triangles, such as those shown in Figure 2, are assigned the same weight. The input image is mapped to this "triangle space" by combining the bicorrelation products corresponding to a given triangle equivalence class.

In principle, a third-order network having been trained on a single target example is capable of recognizing all translated, scaled, and rotated examples of that signature. In practice, however, finite pixel sizes make it difficult to recognize rotated and scaled signatures. To overcome this problem, several techniques are used. First, Spirkovska and $\operatorname{Reid}^{21}$ showed that one can improve performance and simultaneously reduce memory requirements by quantizing the internal angles of the triangles formed. We find it more convenient to quantize the lengths of the triangle sides, having first normalized the longest side to unit length. Perantonis and Lisboa ${ }^{22}$ showed that it was most effective to quantize the largest side of the triangle more agressively. It is also usually beneficial to use a small number of scaled and rotated signatures in training.

Although a single-layer network is sufficient to implement a HONN, Perantonis and Lisboa found that by using an additional (hidden) layer, performance could be improved somewhat. ${ }^{22}$ The resulting three-layer network is trained 


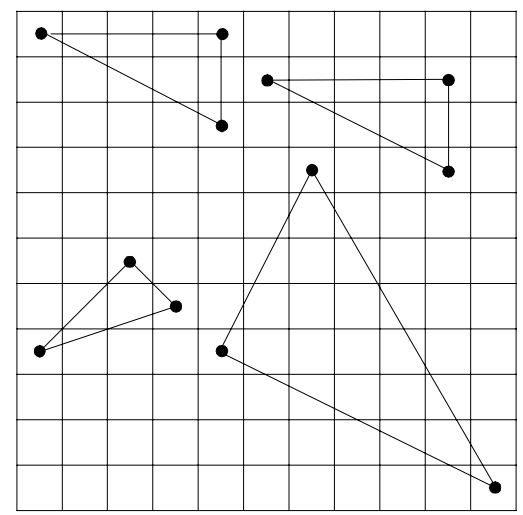

Figure 2. Examples of some pixel triples that define (approximately) similar triangles. Such pixel triples share the same weight in a PSRI network.

using the backpropagation algorithm.

An interesting generalization of the HONN concept is the "functional-link" network of Pao. ${ }^{23}$ In a functionallink network the pixel products are replaced by more general operations (including nonlinear functions) of the form $g_{\ell}\left(x_{i_{1} j_{1}}, x_{i_{2} j_{2}}, \ldots\right)$, viz:

$$
y=f\left(\sum_{\ell=1}^{N} w_{\ell} g_{\ell}(\mathbf{x})\right)
$$

where $\mathbf{x}$ is a vector of inputs to the neuron. It is clear that if we enumerate by $\ell$ the geometrically similar triangle shapes that appear in an image, and if we define the function

$$
g_{\ell}(\mathbf{x})=\sum_{\begin{array}{c}
(n, m, k) \text { form } \\
\text { triangles of shape } \ell
\end{array}} x_{i_{n} j_{n}} x_{i_{m} j_{m}} x_{i_{k} j_{k}}
$$

then the PSRI HONN is equivalent to a functional-link network.

\section{WAVELET PACKET TRANSFORM}

The processing proposed in this paper begin with a wavelet decomposition of the imagery. This step has two benefits. First, it permits us to reject clutter at scales not of interest. In effect, the wavelet decomposition performs the function of a spatial bandpass filter. Nonlinear editing of wavelet coefficients for noise suppression or image compression has been extensively used in the literature. ${ }^{24}$ (See Dohono et al. ${ }^{25}$ for a more recent review.) The use of wavelets for estimating trends is a closely related function and has also received attention including use in a recent paper on biomedical signal processing. ${ }^{26}$ Recently, both techniques were used in a demining context. ${ }^{27}$

A second benefit of the wavelet transform is that it can perform image compression, which further reduces memory requirements. We use 2-D wavelet packets ${ }^{28}$ for this purpose. In a wavelet packet analysis the detail coefficients are fully decomposed, producing output packets with a common size. The approximation coefficients output by a conventional two-dimensional wavelet transform are identical to one of the packets. The compression offered by the wavelet transform provides a dramatic reduction in HONN computational requirements. To see this, note that for a conventional dyadic wavelet transform based on an octave (factor of two) change in scale at each level, the number of pixels output in each packet by a 2 -D level- $K$ transform is reduced by the factor $2^{2 K}$. Thus, the number of operations required to form the third-order HONN bicorrelation products is reduced by the factor $2^{6 K}$ if a single wavelet packet is processed and $2^{4 K}$ if all wavelet packets are processed. In practice, edge effects in the wavelet transform cause the true improvement to be slightly less than these factors.

As an example of the wavelet packet transform, consider the surface-laid M19 mine shown in Figure 3. The transform of this image at level two is shown in Figure 4. The packet in the upper left corner represents the compressed chip, which is useful in subsequent HONN processing. Other chips contain additional information. The third chip (from the left) on the top row is the result of processing equivalent to a high-pass filter in the vertical 


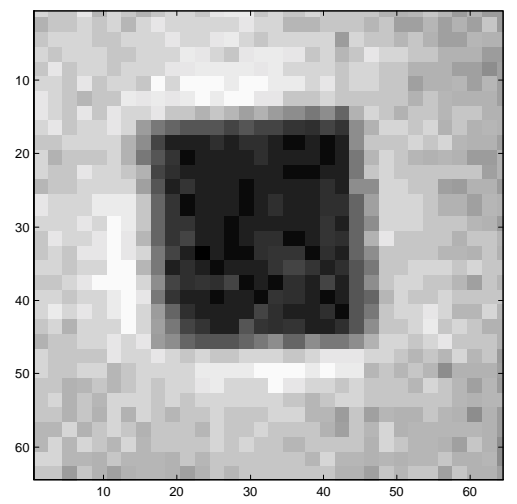

Figure 3. An LWIR image of a surface-laid M19 mine.

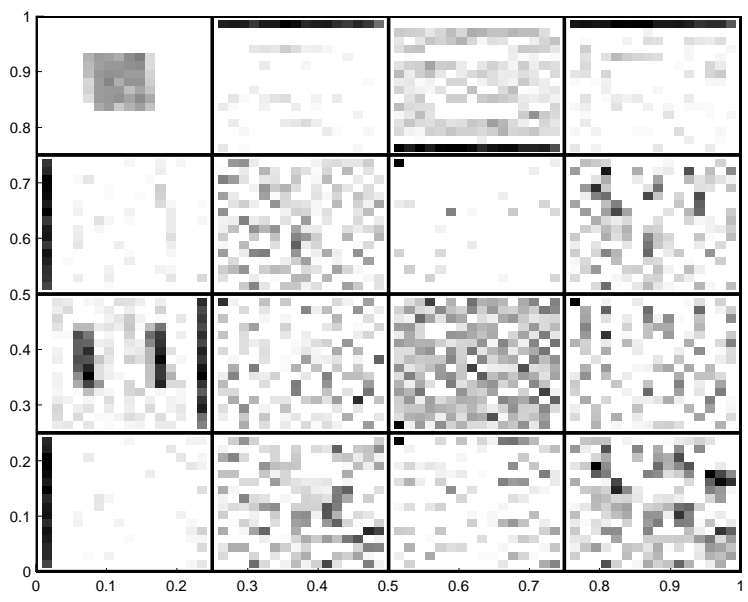

Figure 4. An example of a wavelet packet transform of order two. The pixel count of each output packet is reduced by roughly the factor $2^{4}=16$.

direction. Similarly, the third chip (from the top) in the left-most column is the product of high-pass filtering in the horizontal direction. Other image chips contain primarily small-scale noise-like components that can be neglected.

\section{EXAMPLE RESULTS}

In this section we review three example applications of the wavelet-HONN. In all three cases we apply the algorithm to the detection of surface mines. We describe two examples using simulated images and one using real LWIR data.

The processing used in these examples has both off-line and on-line components. For the off-line processing, one first selects the size of the image chip to be processed by the HONN. In this work we have used $N=16$, for which $N^{6}=1.6 \times 10^{7}$. For an image of this size and a fixed distance quantization, pixel index triples for all similar triangles are identified, and these indices are saved. Next, training data are processed using the on-line operations described below, and the neural network is trained.

On-line processing begins by dividing the image into chips of size $N^{\prime} \times N^{\prime}$, and computing the wavelet packet transform of those chips. The chip size $N^{\prime}>N$ is selected such that a wavelet packet transform at some level $K$ will produce packets of size $N$. In our current implementation only the compressed wavelet packet is used in subsequent processing. The packet is then projected into triangle space by summing the bicorrelation products $x_{i_{k} j_{k}} x_{i_{m} j_{m}} x_{i_{n} j_{n}}$ for indices (determined off-line) comprising similar triangles (cf. Eq. (5)). The resulting sums are the HONN inputs, and the HONN output is used in detection. In our work we used a FFMLP with a single hidden layer and three hidden-layer neurons. The most computationally burdensome part of this process is forming and summing the bicorrelation products. Since this portion of the algorithm is independent of the image and extremely simple, it could be done with specialized hardware. In our implementation all processing is currently done in software. 


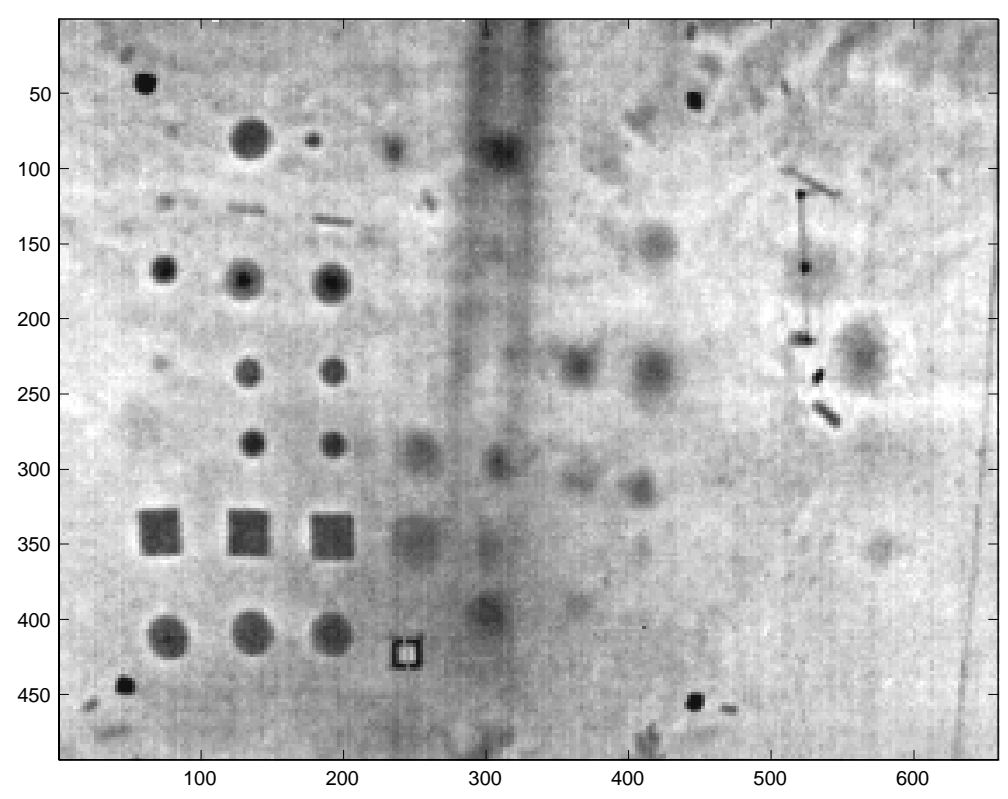

Figure 5. LWIR data used in testing the algorithm.

\subsection{Mine discrimination for well-resolved targets}

A preliminary test of the wavelet-HONN was made using experimental data. IR imagery acquired by E-OIR Measurements, Inc. in July 1998 over a set of mines emplaced at Fort Huachucha, AZ was used for this purpose. An example image is shown in Figure 5. The data were acquired with an Agema 570 LWIR camera looking directly down on the scene. The left half of the scene shows a number of surface-laid anti-tank mines including M19s, M15s and VS1.6s. The center and right half of the image contain a variety of buried mines and clutter artifacts. The prominant smear running through the image top to bottom is a vehicle track. The concentric light and dark squares near the bottom center of the image is a blackbody used for calibration.

We extracted from this scene a number of image chips. The data are shown in Figure 6, where we present 30 examples of surface mines and clutter. In this test we took both square (M19) and circular (M15 and VS1.6) mines to be the targets of interest, which are indicated by the surrounding boxes in Figure 6 . The remaining chips show a variety of clutter objects.

Image chips of $28 \times 28$ pixels were extracted from the data and processed. The naive application of these chips to a $3 \mathrm{D}-\mathrm{HONN}$ would require $28^{6} \approx 5 \times 10^{8}$ input nodes. Using the proposed approach this memory load is reduced greatly. A level-one wavelet packet decomposition produces $16 \times 16$ compressed chips. The projection of these smaller chips onto "triangle space" followed by $5 \%$ distance quantization produces a neural network of 122 inputs.

For this small data set of 30 chips we used the leave-one-out method to train the network and assess its performance. The ROC curve produced through this process is shown in Figure 7. The performance of the network on these data is reasonably good, but the data set is quite small and it contains no rotated targets. The test does, however, offer evidence of scale invariance.

\subsection{Shape discrimination for simulated well-resolved targets}

One potential discriminant for surface anti-tank (AT) mines involves the shape of these targets. In this example we demonstrate the ability of the algorithm to discriminate circular and rectangular shapes. The prototype shapes are shown in Figure 8.

We tested this network with the scaled, rotated, and translated data shown in Figure 9. These data were generated by randomly scaling one of the prototypes by a factor between $3 / 5$ and $5 / 3$. Rotations of $-45^{\circ}$ to $45^{\circ}$ were used, as were random translations. To these images we added spatially white, Gaussian noise to produce a signal to noise ratio of roughly 10:1. In principle, the HONN can be designed to recognize all the exemplars in Figure 9 when 


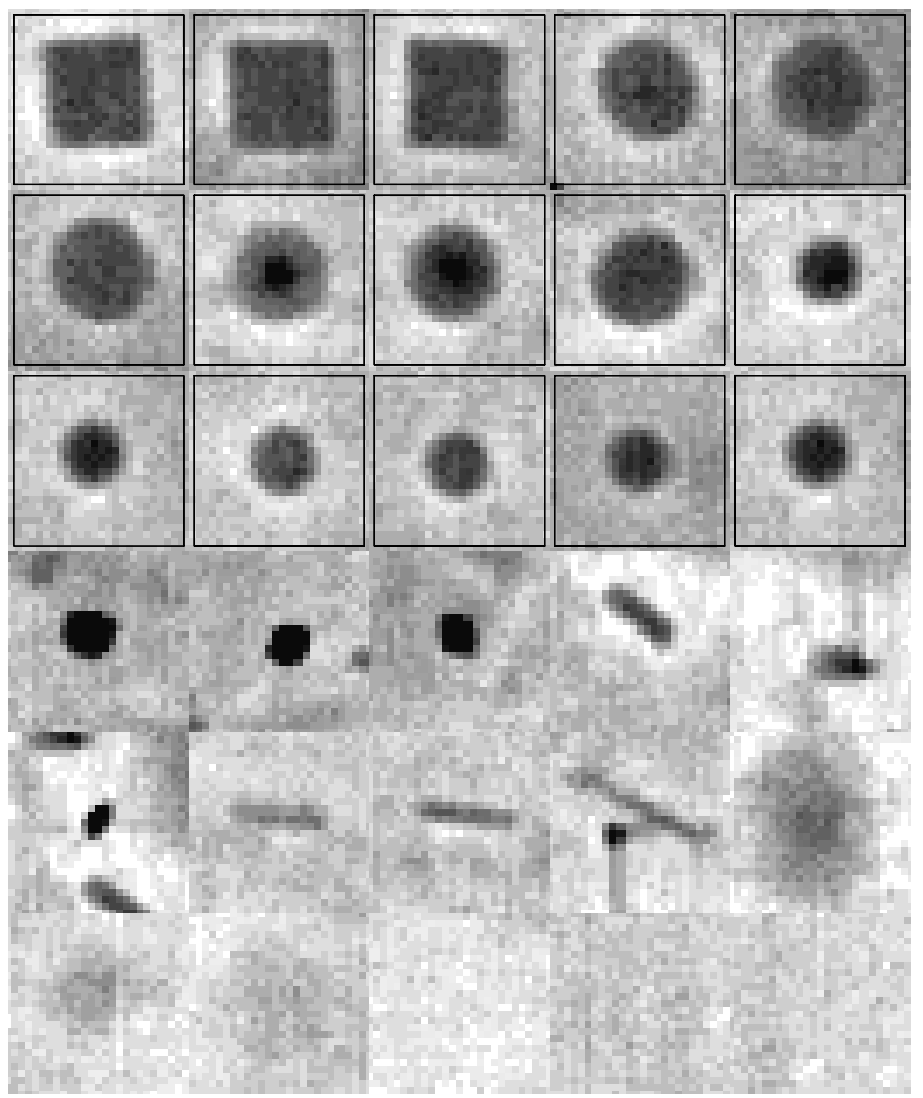

Figure 6. Example target and clutter chips extracted from the LWIR image. The boxed chips are designated targets.

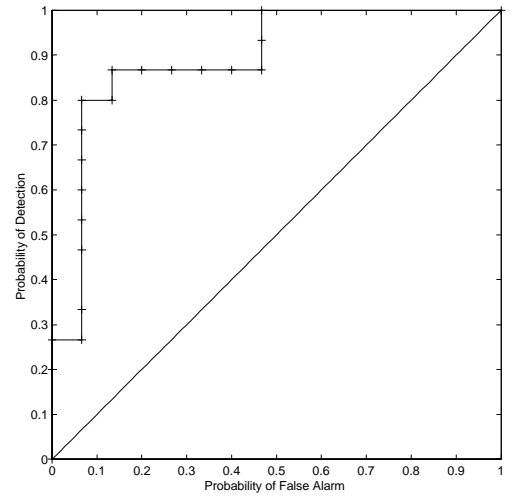

Figure 7. ROC curve generated by the LWIR surface mine data. 


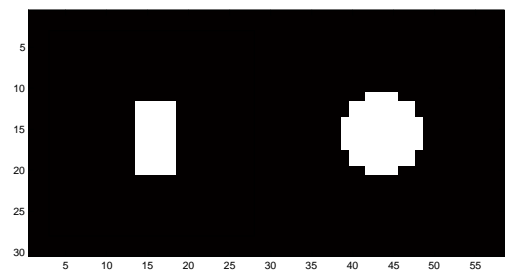

Figure 8. Prototype signatures for rectangular and circular targets.

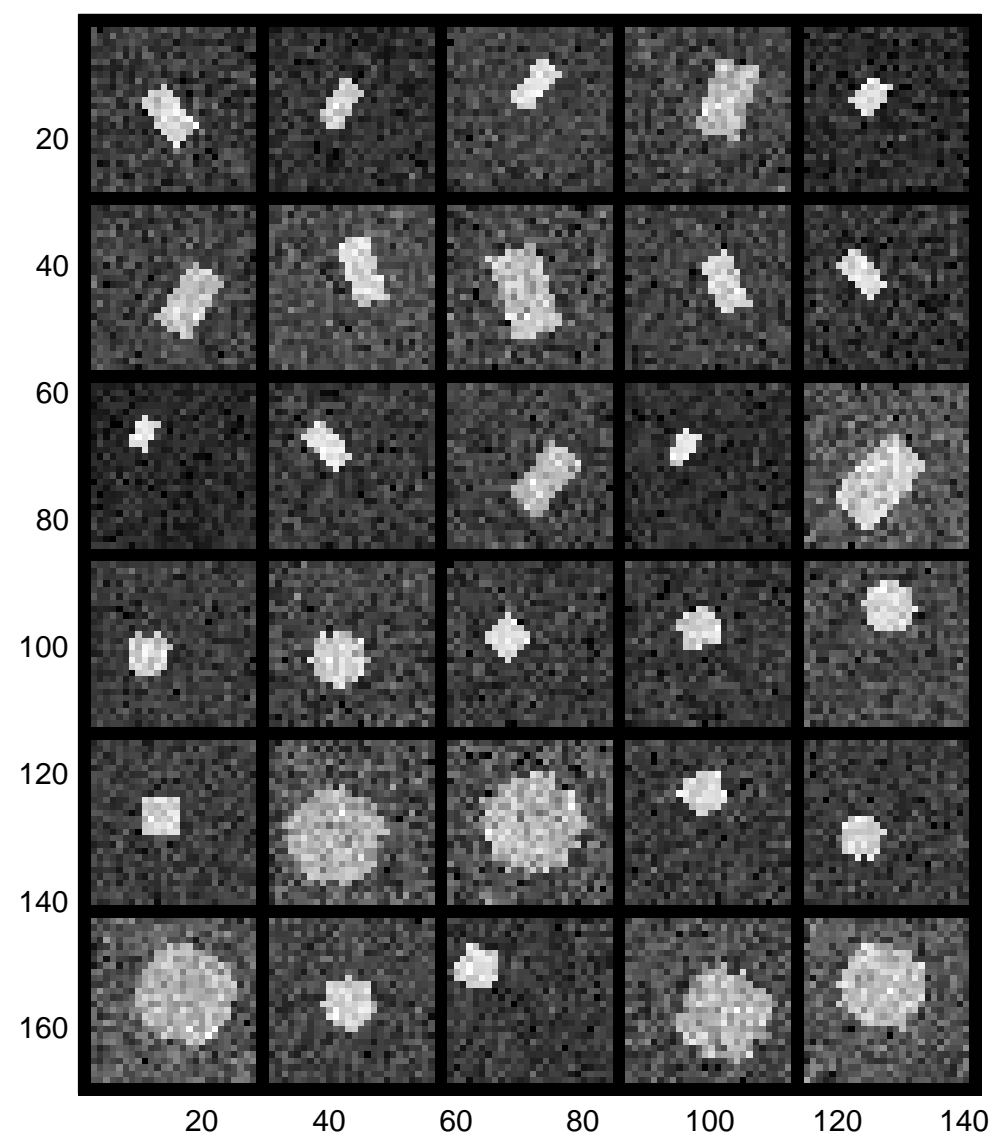

Figure 9. Examples of scaled, translated, and rotated targets used to test the network. The top half of the image contains rectangular targets, and the bottom half contains circular targets. 
trained on the prototypes in Figure 8 only, but finite pixel size effects (noted above) limit this capability. We found performance was improved if we used a small number of scaled and rotated examples to train the network. The network trains quickly (within 10 epochs).

The ROC curve produced for this data set is shown in Figure 10. We find that the performance is good, even though for very small scaling some of the circular targets have a near-square shape.

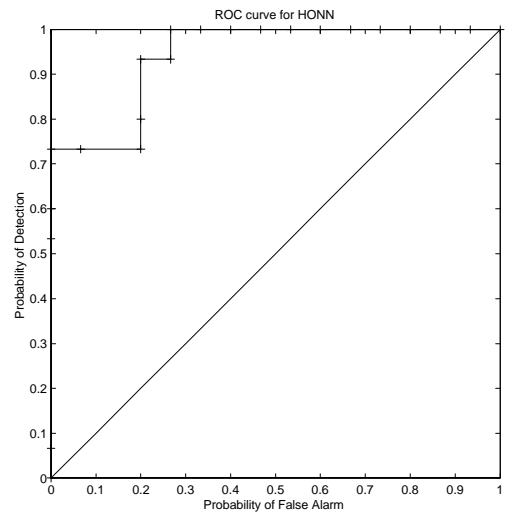

Figure 10. ROC curve generated by classifying scaled, rotated, and translated prototypes.

\subsection{Pattern detection in simulated imagery}

For the final example we consider detection of point targets arranged in a uniform linear array to simulate mine fields. Example data are shown in Figure 11, which contains 30 rotated, translated and scaled scenes. The simulated minefields contain five points in a row with mine-to-mine position errors. The simulated clutter scenes contain four to six points positioned randomly over the scene. A signal-to-noise ratio of roughly 20:1 was employed in these data.

The ROC curve produced by the detection of these targets is shown in Figure 12. Again, relatively good performance is noted, although the data set is small.

\section{CONCLUDING REMARKS}

We have described a new technique for the detection of mines and minefields. The method uses a wavelet packet decomposition as the front-end to a third-order neural network. Use of the wavelet transform and a subsequent projection into "triangle space" greatly reduces the well-known memory problems associated with higher-order neural networks. Tests of the proposed algorithm using simulated imagery and a modest amount of real data have produced encouraging results. More extensive testing using real imagery is planned.

\section{ACKNOWLEDGMENTS}

This project was supported by funds from Duke University under an award from the ARO (the OSD MURI program). The findings, opinions and recommendations expressed therein are those of the authors and are not necessarily those of Duke University or the ARO.

\section{REFERENCES}

1. C. Stewart, "Summary of mine detection research (U)," technical report 1636-TR, U.S. Army Engineer Research and Development Laboratories, Corps of Engineers, Fort Belvoir, VA, May 1960. Volume 1, DTIC AD320124.

2. H. L. Van Trees, Detection, Estimation, and Modulation Theory, vol. 1, Wiley, New York, NY., 1968.

3. I. K. Sendur and B. A. Baertlein, "Techniques for improving buried mine detection in thermal IR imagery," in Detection and Remediation Technologies for Mines and Minelike Targets II, A. C. Dubey, J. F. Harvey, J. T. Broach, and R. E. Dugan, eds., SPIE 3710, pp. 1272-1283, 1999.

4. C. Mehanian and S. J. Rak, "Bi-directional log-polar mapping for invariant object recognition," in Automatic Object Recognition, SPIE 1471, pp. 200-209, 1991. 


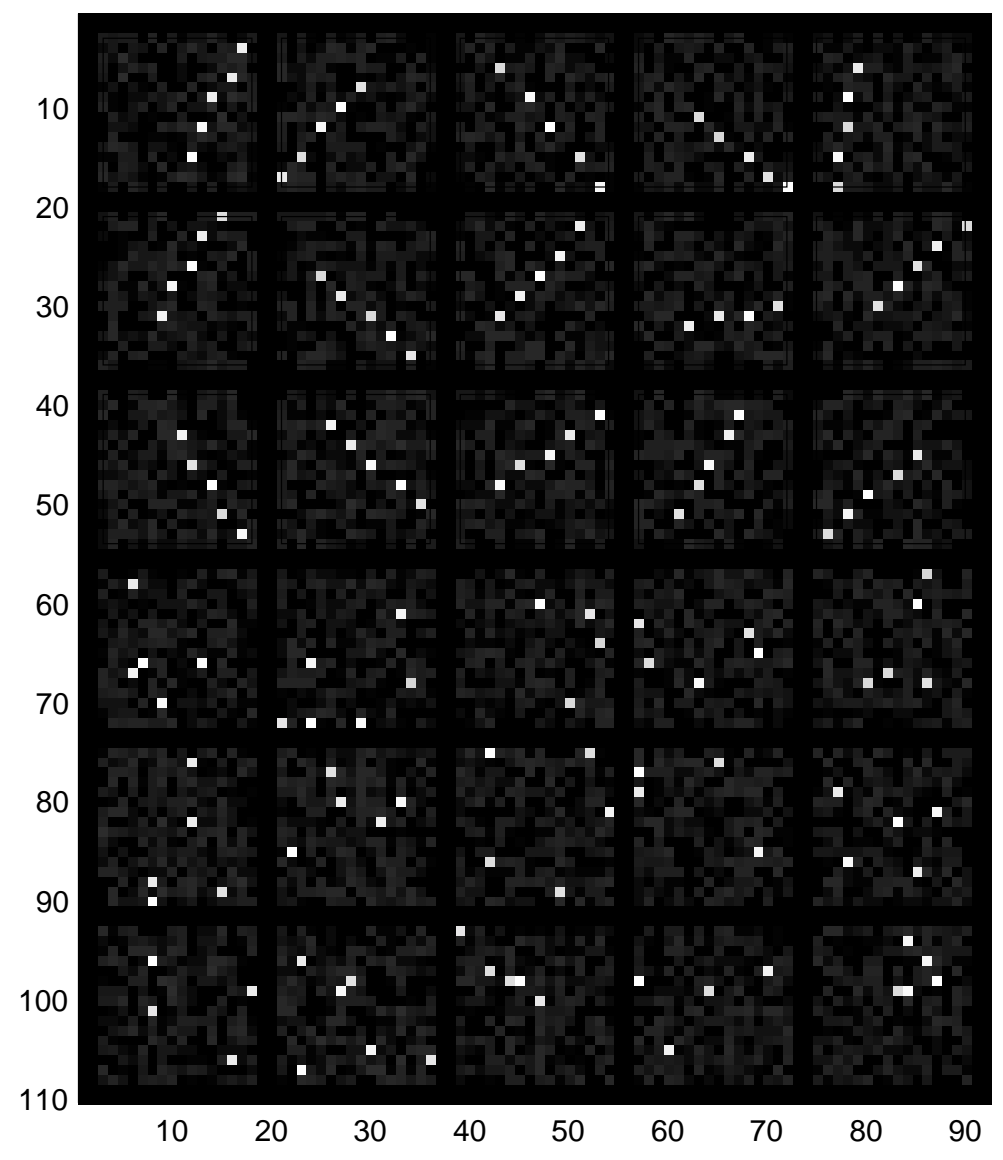

Figure 11. Simulated mine fields and clutter scenes.

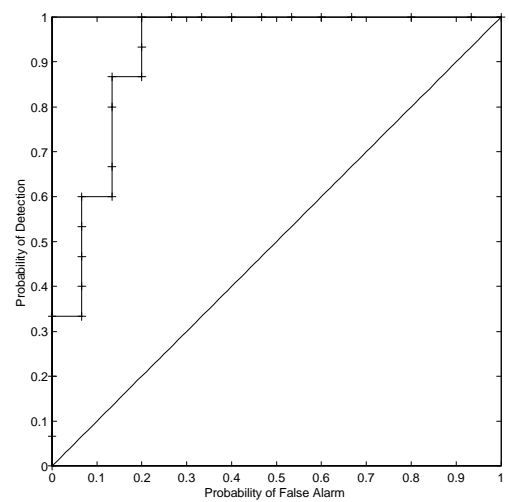

Figure 12. ROC curve generated by the simulated minefields. 
5. D. Casasent and D. Psaltis, "New optical transforms for pattern recognition," Proc. IEEE 65(1), pp. 77-84, 1977.

6. R. A. Altes, "The Fourier-Mellin transform and mammalian hearing," J. Acoust. Soc. Am. 63(1), pp. 174-183, 1978.

7. J. Altmann and H. J. P. Reitbock, "A fast correlation method for scale- and translation-invariant pattern recognition," IEEE Trans. Pattern Analysis and Mach. Intell. PAMI-6(1), pp. 46-57, 1984.

8. P. E. Zwicke and I. Kiss, Jr., "A new implementation of the Mellin transform and its application to radar classification of ships," IEEE Trans. Pattern Analysis and Mach. Intell. PAMI-5(2), pp. 191-199, 1983.

9. B. A. Baertlein and K. F. Casey, "Magnetometric detection of buried objects," in Proceedings of EUROEM, (Bordeaux, France), June 1994.

10. H. H. Szu, X.-Y. Yang, B. A. Telfer, and Y. Sheng, "Neural network and wavelet transform for scale-invariant data classification," Physical Review E 48(2), pp. 1497-1501, 1993.

11. S. H. Yoon, W. E. Alexander, and J. H. Kim, "Scale-invariant shape representation base on the wavelet transform," in Proceedings, SPIE 2232, pp. 142-153, 1994.

12. C. M. Bishop, Neural Networks for Pattern Recognition, Oxford University Press, New York, NY, 1995.

13. S. E. Troxel, S. K. Rogers, and M. Kabrisky, "The use of neural networks in PSRI recognition," in Proc. Joint Int. Conf. Neural Networks, pp. 593-600, (San Diego, CA), July 1988.

14. B. Widrow and R. Winter, "Neural nets for adaptive filtering and adaptive pattern recognition," Computer , pp. 25-39, March 1988.

15. K. Fukushima, "Analysis of the process of visual pattern recognition by the neocognitron," Neural Networks $\mathbf{2}$, pp. 413-420, 1989.

16. K. Fukushima, "A neural network for visual pattern recognition," Computer, pp. 65-75, March 1988.

17. M. Minsky and S. Papert, Perceptrons: An Introduction to Computational Geometry, MIT Press, Cambridge, MA, 1972.

18. Y. C. Lee, G. Doolen, H. H. Chen, G. Z. Sun, T. Maxwell, and H. Y. Lee, "Machine learning using a higher order correlation network," Physica 22D, pp. 276-306, 1986.

19. C. L. Giles and T. Maxwell, "Learning, invariance, and generalization in higher-order neural networks," Applied Optics 26(23), pp. 4972-4978, 1987.

20. Q. Xu, R. M. Inigo, and E. S. McVey, "A combined approach for large-scale pattern recognition with translational, rotational and scaling invariance," in Automatic Object Recognition, SPIE 1471, pp. 378-393, 1991.

21. L. Spirkovska and M. B. Reid, "Higher order neural networks in position, scale, and rotation invariant object recognition," in Fast Learning and Object Recognition, B. Soucek, ed., pp. 153-184, John Wiley, 1992.

22. S. J. Perantonis and P. J. G. Lisboa, "Translation, rotation, and scale invariant pattern recognition by high-order neural networks and moment classifiers," IEEE Trans. Neural Networks 3, pp. 241-251, March 1992.

23. Y.-H. Pao, Adaptive Pattern Recognition and Neural Networks, Addison Wesley, Reading, MA, 1989.

24. R. R. Coifman and M. V. Wickerhauser, "Wavelets and adapted waveform analysis," in Wavelets: Mathematics and Applications, J. Benedetto and M. Frazier, eds., CRC Press, Boca Raton, FL, 1993.

25. D. Dohono, I. M. Johnstone, G. Kerkyacharian, and D. Picard, "Wavelet shrinkage: asymptotia," J. Royal Stat. Soc., Series B 57(2), pp. 301-337, 1995.

26. U. Wiklund, M. Akay, and U. Niklasson, "Short-term analysis of heart-rate variability by adapted wavelet transforms," IEEE Engineering in Medicine and Biology Mag. 16, pp. 113-118, Sept./Oct. 1997.

27. T.-H. Chao, B. Lau, A. Yacoubian, and G. Henderson, "Mine detection using wavelet processing of electro-optic active sensor data," in Detection Technologies for Mines and Minelike Targets, A. Dubey, I. Cindrich, J. Ralston, and K. Rigano, eds., SPIE Proc. 2496, pp. 433-441, 1995.

28. M. V. Wickerhauser, Adapted Wavelet Analysis from Theory to Software, A. K. Peters, Wellesley, MA, 1994. 